\title{
THE MYOPIC EYE OF THE BLACK MOOR GOLDFISH
}

\author{
Stephen S. Easter $J_{R}$ and Peter F. Hitchcock* \\ Division of Biological Sciences, University of Michigan, Ann Arbor, MI 48109, U.S.A.
}

(Received 25 Febrwary 1986)

\begin{abstract}
Optical and anatomical methods were used to establish the refractive state of the eye of the Black Moor variety of goldfish. The eye is strongly myopic. The refractive error in 12 eyes from 6 fish ranged from 89 to $268 \mathrm{D}$.
\end{abstract}

Goldfish Myopia Retina Refractive state

The "Black Moor" strain of goldfish (Carassius auratus) has very large eyes, perhaps the result of high intraocular pressure (Raymond et al., 1984). The retina is larger in area but thinner than that of a common goldfish of the same body length. We have examined the physiological optics of this unusual eye. Earlier studies had established that the common goldfish's eye is approximately hemispheric and emmetropic (Easter et al., 1977). Here, we report that the Black Moor's eye is aspheric and extremely myopic.

The refractive state of the eye was assessed with two optical methods. First, the fundus could not be visualized in an indirect ophthal. moscope (fabricated in the laboratory) in which the fundus of common goldfish can be very well visualized. Subsequent dissection of the eye established that this failure was not attributable to an opacity of the ocular media, lens, or cornea, and must therefore have been due to hypermetropia or myopia. Second, an eye was dissected freshly from an anesthetized fish, immersed in buffer. and the back of the eye was dissected away to expose the vitreous. A high contrast target was placed outside the eye, several centimeters distant, and the back focal plane was located. It lay in the vitreous, millimeters in front of the retina, therefore the eye must be myopic.

The extent of the myopia was estimated anatomically. Our method, developed by Sivak (1974), has been described elsewhere (Easter et

*Present address: Department of Ophthalmology, Kellogg Eye Center. University of Michigan Medical Center, Ann Arbor. MI 48109 , U.S.A. al., 1977). Briefly, the eye is frozen solid and mounted on a frozen stage of a sled microtome. The eye was either removed from an animal killed immediately before mounting, or the entire freshly removed head, with both eyes in situ. was mounted. In all cases, the fish was anesthetized deeply by immersion in $0.1 \%$ tricaine methanesulfonate immediately before freezing. The eye was never exposed to histological fixatives, so it is presumed to have retained its original geometry. The frozen eye was oriented with its pupil facing horizontally and then sectioned. The block face was photographed (Fig. 1) by a camera mounted directly above. A photograph of a millimeter ruler in the position of the eye provided a calibration. The largest section through the center of the lens was analyzed quantitatively in 12 eyes, from 6 fish.

Table 1. Summary of the quantitative data from six pairs of eyes

\begin{tabular}{cccccccc}
\hline Fish & $\begin{array}{c}\text { BL } \\
(\mathrm{cm})\end{array}$ & $\begin{array}{c}\text { LR } \\
(\mathrm{mm})\end{array}$ & $\begin{array}{c}f \\
(\mathrm{~mm})\end{array}$ & $d$ & $\begin{array}{c}\mathrm{EL} \\
(\mathrm{mm})\end{array}$ & $d_{=}$ & $d-d_{\mathrm{s}}$ \\
\hline 237 & 3.2 & 1.1 & 2.6 & 385 & 5.7 & 175 & 210 \\
& & 1.0 & 2.3 & 435 & 6.0 & 167 & 268 \\
238 & 5.5 & 1.6 & 3.7 & 270 & 9.3 & 108 & 162 \\
& & 1.3 & 3.0 & 333 & 9.4 & 106 & 227 \\
239 & 4.4 & 1.3 & 3.0 & 333 & 4.1 & 244 & 89 \\
& & 1.2 & 2.8 & 357 & 7.1 & 141 & 216 \\
240 & 5.4 & 1.3 & 3.0 & 333 & 6.4 & 156 & 177 \\
& & 1.3 & 3.0 & 333 & 8.0 & 125 & 208 \\
241 & 5.2 & 1.2 & 2.8 & 357 & 5.7 & 175 & 182 \\
247 & 11.4 & 1.2 & 2.8 & 357 & 7.2 & 139 & 218 \\
& & 2.2 & 5.1 & 196 & 11.3 & 89 & 107 \\
& & 2.2 & 5.1 & 196 & 12.5 & 80 & 116 \\
\hline
\end{tabular}

Fish: identifying numbers. BL: body length (snout to base of tail). LR: lens radius. $f$ : computed focal length of lens. $d$ : power of lens, in diopters. EL: eye length, distance from lens center to retina along a line to the center of the retina. $d_{\mathrm{e}}$ : strength of the lens, in diopters. for emmetropia at the retinal center $d-d_{i}$ : myopia. in diopters, at the retinal center. 
Both eyes were seldom exactly alike, as Fig. 1 and Table 1 show. Although the radii of the spherical lenses were generally quite similar on the two sides, the eye lengths were not (which suggests that the proximate cause of the defect is probably restricted to the eye). Figure 1 shows the most dissimilar pair of eyes. The focal lengths of the lenses, $f$, were calculated ( $2.32 \times$ lens radius: Charman and Tucker, 1973) and the black semicircles in the two eyes indicate the plane at this distance from the lens center (black dot), at which an object at infinity would be in focus. This is very close to the location of the retina in a normal goldfish with a lens of this size (Easter et al., 1977). But in the Black Moor, the retina lies far behind it. Thus the eye is myopic at all field positions, but particularly at the retinal center. The right column of Table 1 gives the measure of myopia at this position; it ranged from a minimum of 89 to a maximum of $268 \mathrm{D}$.

The margin of the retina, at the ora terminalis, is least myopic. This is incidentally the region at which the new retinal cells are produced. In these animals, as in normal goldfish, the retina grows throughout life by the appositional addition of annuli of cells (P. A. Raymond and P.
Rivlin, personal communication). Thus, the image on the newest retina is probably the least blurred, but as the retina grows and that part near the ora terminalis is displaced centrally, its myopia increases. The ocular enlargement is evident within a few months of hatching (P. A. Raymond, personal communication), and therefore most of the retina has had a blurred image for most of its existence. This eve might be useful as a natural experiment to determine the effects of degradation of image quality on retinal development.

\section{REFERENCES}

Charman W. N and Tucker J. (1973) The optical system of the goldfish eye. Vision Res. 13, 1-8.

Easter S. S. Jr, Johns P. R. and Baumann. L. R. (1977) Growth of the aduit goldfish eye-I: optics. Vision Res. 17, $469-477$.

Hitchcock P. F. (1985) Stretch of the retina contributes to the dendritic field area of gangtion cells in the Black Moor goldfish. Soc. Neurosci. Abstr. 11, 221

Raymond P., Spilman D., Hill R. and Bahn C. (1984) The telescopic eyes of Black Moor goldfish: elevated intraocular pressure and altered aqueous outflow pathways. Invest. Ophthal visual Sci.. Suppl. 25, 282.

Sivak J. G. (1974) The refractive error of the fish eye. Vision Res. 14, 209-213. 
(c)
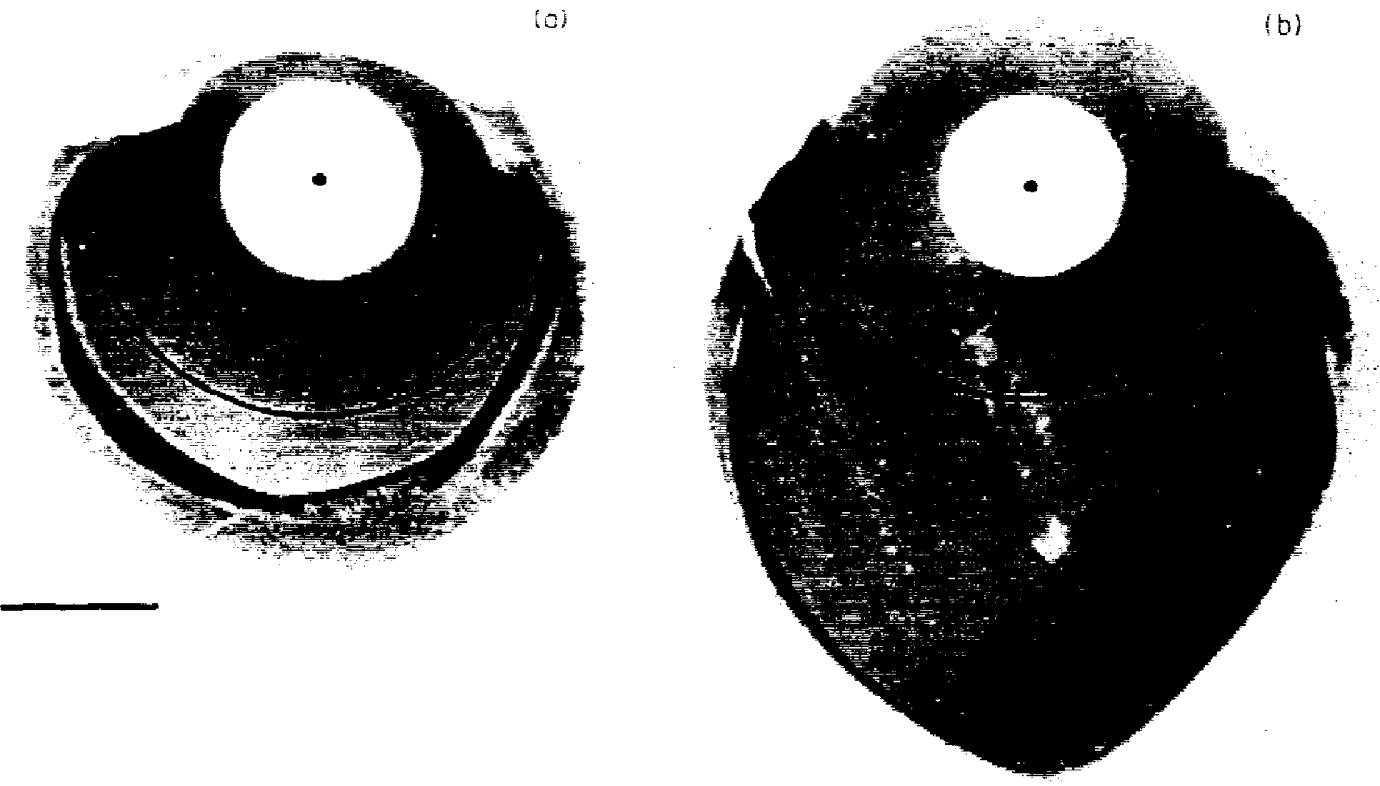

Fig. 1. Two photographs through the centers al the left (a) ard righe (b) eves of the same fish (239) in Table l: The black dot al the lens center is the center for the black are drawn through the vitreus al the piane corresponding to the focal length. The then white strip behind this is the neural retina, in contact with the dark pignemed epuhelium. Suale bar equals 2 mm. 